\title{
Labor Analgesia Reduces the Risk of Postpartum Depression in Women with Single-Term Pregnancy: A Prospective Cohort Clinical Study
}

\section{Li Ren}

First Affiliated Hospital of Chongqing Medical University

\section{Qibin Chen}

First Affiliated Hospital of Chongqing Medical University

Su Min (D 459910606@qq.com )

First Affiliated Hospital of Chongqing Medical University

\section{Fangliang Peng}

First Affiliated Hospital of Chongqing Medical University

\section{Bin Wang}

First Affiliated Hospital of Chongqing Medical University

Jian Yu

First Affiliated Hospital of Chongqing Medical University

\section{Yuxi Zhang}

First Affiliated Hospital of Chongqing Medical University

\section{Research Article}

Keywords: Postpartum depression, labor analgesia, cesarean section

Posted Date: December 2nd, 2020

DOl: https://doi.org/10.21203/rs.3.rs-109373/v1

License: (c) (i) This work is licensed under a Creative Commons Attribution 4.0 International License. Read Full License 


\section{Abstract}

Background: Postpartum depression (PPD) is a frequent mental disorder during the first year after delivery. Previous studies reported that patients with labor analgesia revealed lower incidence of PPD compared to those without. However, most pregnant women gave birth by labor analgesia or cesarean section in China, it is still unclear that whether there is a difference of the effects of labor analgesia and cesarean section on PPD.

Methods: A single-centered, prospective cohort clinical study was designed. One hundred ninety-eight patients with single pregnancy at full term were allocated to receive epidural labor analgesia (group LA) or cesarean section with epidural anesthesia (group CS) according to the maternal preference of delivery and professional suggetions of obstetricians and anesthesiologists. PPD was assessed with Edinburgh Postnatal Depression Scale (EPDS) scores $\geq 10$ at 6 weeks postpartum. Maternal and neonatal variables in the perinatal period were recorded. Multivariate logistical regression analysis was conducted to evaluate the associated factors of PPD.

Results: 11 patients in group LA and 26 patients in group CS was diagnosed as PPD. The incidence of PPD in group LA was lower than group CS $(P=0.011)$. Eight potential predictor factors (labor analgesia, family income, duration of labor, antenatal depression, perinatal classes attending, neonate of male, EPDS scores at 3 days postpartum and adverse event at 6 weeks postpartum) were correlated with PPD. And it was reported in the final model that labor analgesia was the protective factor of PPD, however, high family income and EPDS scores at 3 days postpartum were associated with an increased risk of PPD.

Conclusions: Patients with epidural labor analgesia were associated with lower incidence of PPD compare to those with cesarean section with epidural anesthesia. Epidural labor analgesia, family income and EPDS scores in the early postpartum period were the independent predictors of PPD.

Trial registration: This clinical trial was registered at the Chinese Clinical Trial Registry (http://www.chictr.org.cn/index.aspx). Number: ChiCTR1900020510. Registered on: 3/1/2019.

\section{Background}

During pregnancy, women experience substantial changes in physiology and psychology that may be a great source of concern. Yet, quite often, recognition of mental disorders is not given adequate attention in this population. Postpartum depression (PPD), defined as psycholespy during the first year after labor, is a frequent mental disorder related to childbearing[1]lt was reported that $14 \%$ of mothers in the USA experienced PPD [2]Relatively, the prevalence of PPD in China was reported to be $11.8 \%$ [3]Mothers with PPD have poor social and psychological functions and are more likely to exhibit behaviors, which could compromise parent-child attachment[4]In addition, PPD can induce a mother's suicide, which has been established as the main cause of death during the first postpartum year[5]PPD has also been associated with negative effects on infants and families, while previous studies have reported close correction of 
PPD in mother and paternal postpartum depression [6]Nevertheless, the etiology of PPD remains poorly understood, and effective preventive interventions are urgently needed.

Many risk factors, such as neuroticism[7]and stress of labor, have been reported to be involved in the development of PPD [8]Nowadays, three types of labor are practiced in clinical: natural childbirth (vaginal birth with no epidural analgesia), labor analgesia (vaginal birth with epidural analgesia), and cesarean section (conducted with intraspinal or general anesthesia). Due to intensive birth pain, pregnant women prefer labor analgesia instead of natural childbirth[9]The medical care burden related to childbirths in huge populations such as China is expectedly high. Since it requires less time, the cesarean section has gradually become the first choice for birth in many cities. Previous studies have reported relatively high cesarean section rates in China, reaching up to 32.7\% 50\%[10-12] Labor analgesia and cesarean section have become the main birth practices in China. Although labor pain can be relieved with the use of anesthesia and analgesia regardless of cesarean section or labor analgesia, the incidence of bleeding, placenta previa in a second pregnancy, the intensity of postpartum pain and other complications have been reported to be higher among women undergoing cesarean section compared to those receiving labor analgesia[13]Therefore, the psychological changes are not similar among patients who received labor analgesia or cesarean section, which could potentially affect PPD. However, numerous studies have only focused on the differences in PPD among subjects who experienced vaginal birth with or without labor analgesia $[14,15]$ It still remains unclear whether labor analgesia and cesarean section could affect PPD.

The aim of this prospective cohort clinical study was to investigate the effects of labor analgesia and cesarean section on PPD using the Edinburgh postnatal depression scale (EPDS) scores at 6 weeks after birth. Moreover, we further explored the predicted factors of PPD during the perinatal period.

\section{Methods}

The study was conducted in the First Affiliated Hospital of Chongqing Medical University between January 2019 and November 2019. The protocol was approved by the ethics committees of the First Affiliated Hospital of Chongqing Medical University (No. 2018-100). This study was registered at the Chinese Clinical Trial Registry on 3/1/2019, number: ChiCTR1900020510.

\section{Subjects}

Full-term pregnant women with singleton aged 18 to 45 years old undergoing epidural labor analgesia or elective cesarean section with epidural anesthesia were recruited. Participants were excluded if they were diagnosed with mental illness, cesarean section with general anesthesia, contradiction for epidural anesthesia (including coagulation disorder, infection at the puncture site, lumbar injury), local anesthetics allergy, and severe illness complicating pregnancy (including severe hypertension, diabetes, or other severe heart, hepatic, renal disorders). Participants were also excluded if they were bleeding over $800 \mathrm{ml}$ or had a cardiac arrest during birth. To reduce the bias of accidental psychological fluctuation, patients with 
incomplete epidural anesthesia who needed general anesthesia or those who due to failure of natural childbirth with labor analgesia, turned to cesarean section, were not included in this study.

\section{Groups}

On admission to the hospital, all eligible patients were informed about the study, and consent was obtained from each patient. Information about the advantage and disadvantage of labor analgesia and cesarean section were also provided. Combined with the professional suggestions from obstetricians, each patient made her own decision to have labor analgesia (group LA) or cesarean section (group CS). Subjects in group LA received epidural labor analgesia in the delivery room before birth, while subjects in group CS received epidural anesthesia in the operation room before surgery.

\section{Epidural labor analgesia}

The cervix was checked by a midwife or obstetrician every 4 hours during the latent phase of the first stage. When the cervix was dilated to $2 \mathrm{~cm}$ or more, the epidural labor analgesia was conducted by a senior anesthesiologist (QB Chen). The pregnant woman was placed in a lateral position, and L2-L3 interspace was chosen for puncture and catheterization. An initial loading dose of $5 \mathrm{ml}$ of $1 \%$ lidocaine was administrated. After confirming anesthesia's effect and ruling out the adverse event (spinal anesthesia and local anesthetic intoxication), patient-controlled epidural analgesia (PECA) pump was connected to the catheter. The formula of PCEA included $150 \mathrm{mg}$ ropivacaine and $0.25 \mathrm{mg}$ fentanyl with saline added up to a volume of $150 \mathrm{ml}$ in total. The patient received a loading dose of $8 \mathrm{ml}$ when the connection was completed, followed by an infusion rate of $8 \mathrm{ml} / \mathrm{h}$, with a bolus of $8 \mathrm{ml}$ and a lock time of $20 \mathrm{~min}$. The anesthesiologist solved any side effects related to the epidural analgesia. Epidural labor analgesia was canceled when the birth was completed.

\section{Epidural anesthesia for elective cesarean section}

Epidural anesthesia was conducted by a senior anesthesiologist (B Wang) for each patient who needed an elective cesarean section. Firstly, Vein puncture and catheterization were conducted by a nurse when the patient was sent to the operation room. Similarly, the patient was placed in a lateral position, and L1L2 interspace was chosen for puncture and catheterization. An initial loading dose of $3 \sim 5 \mathrm{ml}$ of $2 \%$ lidocaine was administrated to test the effect of anesthesia and whether there were adverse events related to epidural anesthesia. A dose of $10 \sim 15 \mathrm{ml}$ of $2 \%$ lidocaine with 1:200,000 epinephrine was subsequently administrated. If the block was insufficient, $5 \sim 7 \mathrm{ml}$ of $1 \%$ ropivacaine was added via the epidural catheter. The surgery was conducted by a senior obstetrician (FL Peng) when the level of anesthesia ranged from T6 to S3. Cesarean section was conducted through a transverse lower uterine segment incision. The uterine wound was closed in the continuous absorbable suture, and oxytocin or ergometrine was used if necessary. The skin incision was closed with nonabsorbable stitches, which were removed on day $7 \sim 10$ postoperatively. Also, all patients received patient-controlled intravenous analgesia (PCIA) at the end of surgery. The formula of PCIA included tramadol $800 \mathrm{mg}$, nefopam $40 \mathrm{mg}$ 
with saline added up to a volume of $80 \mathrm{ml}$ in total. The parameters were set as follows: $5 \mathrm{ml}$ for a loading dose, $1 \mathrm{ml} / \mathrm{h}$ for infusion rate, $5 \mathrm{ml}$ for bolus dose, and $20 \mathrm{~min}$ for the lock time.

\section{General data collection}

The demographic characteristics, including age, body mass index (BMI), education background, and the information on the present pregnancy (including gravidity and parity, perinatal classes about childbirth, present obstetrical and gynecological disease, disease complicating pregnancy) were collected by using a designed information form after receiving written consent from the subject. Perinatal classes were educational courses prepared for expectant mother and father conducted by hospital or birth-feeding institutions, which provided information on perinatal health care, the process of birth, infant feeding, postpartum recovery, and parenting. In addition, the baseline of the numerical rating scale (NRS) scores was collected for each patient. In group LA, the baseline of pain was assessed by NRS before starting epidural labor analgesia, while the baseline NRS scores were evaluated before starting epidural anesthesia in group CS. Besides, antenatal depression was also evaluated using the Edinburgh Postnatal Depression Scale (EPDS). The EPDS is a 10-item questionnaire with maximum scores 30 , which is widely used for the detection of postnatal depression $[14,16]$ Recently, the EPDS has been shown to be an effective screening tool for antenatal depression, where a cutoff score of 10 or 11 is used for the identification of antenatal depression[17, 18]In the present study, antenatal depression was defined as an EPDS score of 10 or higher before birth.

\section{Postpartum follow-up}

The EPDS scores were recorded at 3 days and 6 weeks after birth. PPD was diagnosed as EPDS score $\geq$ 10 at 6 weeks after birth. Additionally, the pain at rest and in motion was assessed by NRS scores at 1, 2, 3 days, and 6 weeks after birth. Pain at rest was defined when the patient was lying in a supine position in bed, and pain in motion was rated when the patient was coughing or walking on level ground. Any adverse event was recorded within 3 days and 6 weeks after birth. Duration of labor and neonatal variables (neonatal gender, body weight, Apgar score 1, 5, $10 \mathrm{~min}$ after birth) were also followed. Duration of labor was defined as the time from the beginning of regular contractions (lasting more than 30s with an interval of $5 \sim 6$ minutes) to the end of birth in group LA, while the duration was calculated as operation time in group CS. In China, mothers are traditionally expected to rest indoors for one full month after birth, so the data on companion during this time (professional maid, patient's parents or/and parents-in-law) and infant feeding choices (formula milk or/and breast milk) were also recorded.

\section{Sample calculation and statistical analysis}

According to the previous report, we assumed that that the incidence of PPD would be $20 \%$ for subjects who underwent cesarean section and $14 \%$ for pregnant women who received labor analgesia[14, 19]The statistical power of $80 \%$ at the 0.05 significance lever required 100 subjects in each group, and the final sample size was 110 subjects considering a $10 \%$ dropout rate. The sample size was calculated by two independent proportions of power analyses of PASS 11. 
All data were expressed as mean \pm standard deviation (normal distribution data) or median (interquartile range) (non-normal distribution data) for continuous variables and total number (percent frequency) for categorical variables. The results of continuous variables were compared using the $t$-test (normal distribution data) or Wilcoxon rank-sum test (non-normal distribution data). The chi-square test was used to analyze the results of categorical variables. Fisher's exact test was used for categorical variables when the number of events was less than 5 . The predicted factor of PPD was assessed with multivariate logistic regression analysis. All the perinatal variables in this present study, including maternal and neonatal factors, were included in the univariate analysis to screen for potentially predictive factors. To avoid missing some potential important variables, factors with a $P$-value $\leq 0.1$ in univariate analysis were defined as candidate variables[20]In consideration of a substantial bias of estimated regression coefficients generated using the stepwise method[21]all the potential variables were included in the multivariable model to determine the predictive factors by using the method of enter procedure. $P$-value < 0.05 was considered as statistically significant.

\section{Results}

Among a total of 294 screened patients, 74 were excluded for not meeting the criteria or refusing to participate. The remaining 220 patients were allocated to group CS or group LA according to the professional evaluation of obstetricians and anesthesiologists. Thirteen subjects in group LA and nine subjects in group CS did not complete the follow-up. Ultimately, 97 patients in group LA and 101 patients in group CS were included in the final analysis (Figure 1).

\section{Baseline characteristics of eligible patients}

The baseline characteristics of the subjects are shown in Table 1. Pregnant women in group CS were much older and had higher BMI compared with group LA ( $P \otimes 0.001)$. Additionally, gravidity and parity in group CS were increased compared to group LA $(P \otimes 0.001)$. More patients in group LA attended the perinatal classes during pregnancy $(P \otimes 0.001)$. Besides, the baseline NRS scores were higher in group LA than in group CS (Pख0.001). Other parameters were not significantly different between group CS and group LA $(P \otimes 0.05)$.

\section{Difference of perinatal variables within 6 weeks follow-up}

A significant difference was found in EPDS scores at 3 days after birth between group LA and group CS $(P=0.003)$. Similarly, EPDS scores at 6 weeks after birth were also lower in group LA than in group CS $(P=0.014)$. Moreover, 11 in group LA and 26 patients in group CS were diagnosed with PPD at 6 weeks after birth, and the incidence of PPD in group LA was lower than in group CS $(P=0.011)$ (Table 2).

The patients who underwent cesarean section spent much less time giving birth ( $P \otimes 0.001)$. NRS scores, which were recorded for all subjects in this study, were higher in group CS than in group LA at rest at 3 days $(P=0.001)$, in motion at 1 day $(P=0.002)$ and at 6 weeks $(P[0.001)$ after birth; however, no significant difference was found at other time points within the 6 weeks follow-up ( $P \bowtie 0.05)$. Additionally, 29 cases in 
group CS and 18 cases in group LA had adverse events at 3 days after birth, and the incidence of adverse events was significantly lower in group CS $(P=0.046)$. However, there were no differences in the occurrence of adverse events at 6 weeks after birth $(P=0.203)$. There was also no significant difference in feeding modalities between the two groups $(P=0.099)$. However, a significant difference was found in the comparison of the main companion during maternal rest indoor at one month after giving birth $(P=0.01)$ (Table 2).

In addition, variables of neonates were also recorded. There were no differences between the two groups in the gender, body weight or Apgar scores at 1, 5, and 10 minutes after birth (P凶0.05)(Table 3).

\section{Factors associated with PPD}

Univariate analysis was used to screen for potentially predictive factors of PPD. Thirty-three variables were included, and 8 variables were selected as candidate variables, including labor analgesia, family income, duration of labor, antenatal depression, perinatal classes attending, neonate of male, EPDS at 3 days after birth, adverse event at 6 weeks after birth. All 8 variables were included in the multivariate logistic regression model using the enter method. Finally, three independent predictors of PPD were identified: labor analgesia (OR $0.049,95 \% \mathrm{Cl} 0.003-0.955, P=0.047$ ), family income (OR $7.267,95 \% \mathrm{Cl} 1.65$ $32.014, P=0.009$ ), and EPDS at 3 days after birth (OR 1.275, 95\% Cl 1.14-1.426, $P \otimes 0.001$ ). Hosmer-

Lemeshow test suggested the model to be a good fit $(\chi 2=9.758, \mathrm{df}=8, P=0.282)$ (Table 4$)$.

\section{Discussion}

In the present study, we found that pregnant women who received labor analgesia had a lower incidence of PPD when compared to those who underwent cesarean section. Labor analgesia was associated with an independent decreased risk of PPD; however, high family income and EPDS scores at three days after giving birth were associated with an increased risk of PPD for pregnant women.

The identified relationship between pain and depression has already been reported in the general population[22]Naturally, it was logical to speculate that labor pain experienced by obstetric patients would contribute to PPD. The intensity of labor pain has been related to a mood disorder in the early postpartum period[23]Lim et al also reported that improvement in labor pain was a significant predictor of PPD[24]Therefore, accumulative evidence suggests that patients who used labor analgesia for pain control had a lower incidence of PPD than those who did not $[14,24,25]$ In the present study, we found that labor analgesia was associated with a decreased risk of PPD when compared with the cesarean section, although no significant association was revealed between perinatal pain and PPD. A reasonable explanation is that the birth modes, i.e., vaginal birth, possibly make a difference in PPD. This assumption has been verified by previous studies reporting that risk of acquiring PPD was lower in mothers who underwent vaginal birth compared to those undergoing cesarean section[26-28]In addition, it was reported that changes in treatment pain were more important to patients than the pain itself[29]and patients with the expectation-to-reality matching of perinatal pain control possibly revealed a lower risk of PPD [24] In our study, most patients in the two groups experienced mild pain, which potentially matched 
their expectations, although the NRS scores were higher in the CS group than in group LA at some time points. Consequently, two factors, including the birth mode and pain control, should be paid special attention when considering the effect of labor analgesia on PPD. Also, further studies are needed to illuminate the influence of different modes of birth and ways of perinatal pain control on PPD.

Family income has been verified to be correlated with PPD, i.e., low family income is regarded as a risk factor for PPD[30]Interestingly, in the present study, subjects with high family income were susceptible to PPD. Patients with low family income usually have to deal with more difficulties in life and more worry about the future. However, this does not necessarily mean that patients with high family income lead a carefree life exclusively as they may lead to more interpersonal, social, and business stress. A previous study reported that children who lived in wealthy families suffered from more serious depression and anxiety disorders[31]In this study, the average family income reached approximately 8,000 CNY per month (a relatively comfortable level of living in Chongqing, China), which suggested that most participants did not suffer from severe financial strain, thus potentially highlighting the negative influence of high family income.

EPDS is a self-report, user-friendly questionnaire that was used to evaluate PPD in the present study. However, it should be stressed that the EPDS is just a screening PPD scale used by non-psychiatrists instead of professional's mental diagnosis tool. PPD has been assessed by EPDS in many different studies, including ours, mainly because it has an estimated $80 \%$ sensitivity for a diagnosis of depression[32] Growing evidence has suggested that high peripartum depression scores are strong predictors of PPD $[33,34]$ In this study, we found that high EPDS scores at 3 days postpartum were an independent risk factor of PPD. Most mothers in China tend to stay in hospitals within 3 days after birth, which can directly help doctors identify the high-risk patients of PPD early, thus prompting timely intervention to prevent PPD. In the present study, the time point to assess PPD was chosen at 6 weeks postpartum, which was consistent with numerous previous studies[35-37]Nonetheless, other time points have also been reported[38-40]At present, there is no universally accepted time point for PPD assessment, and it still remains unclear whether different assessment time points could influence PPD results.

The present study has several limitations. First, this is a prospective cohort study; thus, randomization and blinding could not be conducted. Accordingly, the results could be inevitably influenced by bias. Second, PPD was only diagnosed with EPDS; no other evaluation scale for PPD was used in this study, nor was there any professional diagnosis of psychiatrists. Third, the participants were visited within 6 weeks after birth, which means that the follow-up duration was not long enough, and the long-term effects of labor analgesia and cesarean section could not be investigated. Fourth, the baseline characteristics were not compared between the two groups in this clinical trial; therefore, the causal relationship between birth mode and outcomes could not be confirmed.

\section{Conclusions}


Compared to the cesarean section, labor analgesia was more effective in reducing the incidence of PPD. Labor analgesia was regarded as a protective factor of PPD; however, high family income and EPDS scores at 3 days postpartum were associated with an increased risk of PPD. Nonetheless, the clinical significance of labor analgesia in PPD needs to be further verified.

\section{Abbreviations}

BMI: body mass index; CNY: Chinese yuan; EPDS: Edinburgh postnatal depression scale; NRS: numerical rating scale; PCEA: patient-controlled epidural analgesia; PCIA: patient-controlled intravenous analgesia; PPD: postpartum depression;

\section{Declarations}

\section{Acknowledgements}

The authors gratefully acknowledge Dr. Jianmei Chen, associate professor, of the Department of Psychiatrics, the First Affiliated Hospital of Chongqing Medical University, for providing psychiatric consultation.

\section{Authors' contributions}

Li Ren contributed to design and conduct of the study, data analysis, and editing of manuscript. Qibin Chen, Fangliang Peng and Bin Wang participated in conduct of the study. Su Min contributed to experimental design, data interpretation and editing of manuscript. Jian Yu and Yuxi Zhang participated in data collection, data analysis. All authors read and approved the final manuscript prior to submission.

\section{Funding}

This work was supported by the National Natural Science Foundation of China. (No. 81901377)

\section{Availability of data and materials}

All data generated or analysed during this study are included in this published article.

\section{Ethics approval and consent to participate}

The protocol was approved by the ethics committees of the First Affiliated Hospital of Chongqing Medical University on Oct 15th, 2018 (No. 2018-100). All methods were performed in accordance with the Declaration of Helsinki and relevant guidelines, informed consent was obtained from all participants.

\section{Consent for publication}

Not applicable 


\section{Competing interests}

The authors declare that they have no competing interests

\section{Author details}

3. Department of Anesthesiology, the First Affiliated Hospital of Chongqing Medical University, Chongqing, China.

4. Department of Obstetrics, the First Affiliated Hospital of Chongqing Medical University, Chongqing, China.

\section{References}

1. Bell AF, Andersson E. The birth experience and women's postnatal depression: A systematic review. Midwifery. 2016;39:112-23.

2. Wisner KL, Sit DK, McShea MC, et al. Onset timing, thoughts of self-harm, and diagnoses in postpartum women with screen-positive depression findings. JAMA Psychiatry. 2013;70(5):490-8.

3. Wang YY, Li H, Wang YJ, et al. Living with parents or with parents-in-law and postpartum depression: A preliminary investigation in China. J Affect Disord. 2017;218:335-8.

4. Meaney MJ. Perinatal Maternal Depressive Symptoms as an Issue for Population Health. Am J Psychiatry. 2018;175(11):1084-93.

5. O'Hara MW. Postpartum depression: what we know. J Clin Psychol. 2009;65(12):1258-69.

6. Goodman JH. Paternal postpartum depression, its relationship to maternal postpartum depression, and implications for family health. J Adv Nurs. 2004;45(1):26-35.

7. Iliadis $\mathrm{SI}$, Koulouris P, Gingnell M, et al. Personality and risk for postpartum depressive symptoms. Arch Womens Ment Health. 2015;18(3):539-46.

8. Wisner KL, Stika CS, Clark CT. Double duty: does epidural labor analgesia reduce both pain and postpartum depression. Anesth Analg. 2014;119(2):219-21.

9. Shatalin D, Weiniger CF, Buchman I, Ginosar Y, Orbach-Zinger S, loscovich A. A 10-year update: national survey questionnaire of obstetric anesthesia units in Israel. Int J Obstet Anesth. 2019;38:8392.

10. Hou L, Li G, Zou L, et al. [Cesarean delivery rate and indications in mainland China: a cross sectional study in 2011]. Zhonghua Fu Chan Ke Za Zhi. 2014;49(10):728-35.

11. Li HT, Luo S, Trasande L, et al. Geographic Variations and Temporal Trends in Cesarean Delivery Rates in China, 2008-2014. JAMA. 2017;317(1):69-76.

12. Liu Y, Wang X, Zou L, Ruan Y, Zhang W. An analysis of variations of indications and maternal-fetal prognosis for caesarean section in a tertiary hospital of Beijing: A population-based retrospective cohort study. Medicine (Baltimore). 2017;96(7):e5509. 
13. Ben-Meir A, Schenker JG, Ezra Y. Cesarean section upon request: is it appropriate for everybody. J Perinat Med. 2005;33(2):106-11.

14. Ding T, Wang DX, Qu Y, Chen Q, Zhu SN. Epidural labor analgesia is associated with a decreased risk of postpartum depression: a prospective cohort study. Anesth Analg. 2014;119(2):383-92.

15. Nahirney M, Metcalfe A, Chaput KH. Administration of epidural labor analgesia is not associated with a decreased risk of postpartum depression in an urban Canadian population of mothers: a secondary analysis of prospective cohort data. Local Reg Anesth. 2017;10:99-104.

16. Cox JL, Holden JM, Sagovsky R. Detection of postnatal depression. Development of the 10-item Edinburgh Postnatal Depression Scale. Br J Psychiatry. 1987;150:782-6.

17. Castro E Couto T, Martins Brancaglion MY, Nogueira Cardoso M, et al. What is the best tool for screening antenatal depression. J Affect Disord. 2015;178:12-7.

18. Al-Hejji Z, Al-Khudhair M, Al-Musaileem M, Al-Eithan M. Prevalence and associated risk factors of antenatal depression among women attending antenatal clinics in primary health care centers in the Ministry of Health in Al-Ahsa City, Saudi Arabia. J Family Med Prim Care. 2019;8(12):3900-7.

19. Xie RH, Lei J, Wang S, Xie H, Walker M, Wen SW. Cesarean section and postpartum depression in a cohort of Chinese women with a high cesarean delivery rate. J Womens Health (Larchmt). 2011;20(12):1881-6.

20. Zhang BF, Wang PF, Fei C, et al. Perioperative Deep Vein Thrombosis in Patients With Lower Extremity Fractures: An Observational Study. Clin Appl Thromb Hemost. 2020;26:1076029620930272.

21. Steyerberg EW, Eijkemans MJ, Habbema JD. Stepwise selection in small data sets: a simulation study of bias in logistic regression analysis. J Clin Epidemiol. 1999;52(10):935-42.

22. Bair MJ, Robinson RL, Katon W, Kroenke K. Depression and pain comorbidity: a literature review. Arch Intern Med. 2003;163(20):2433-45.

23. Boudou M, Teissèdre F, Walburg V, Chabrol H. [Association between the intensity of childbirth pain and the intensity of postpartum blues]. Encephale. 2007;33(5):805-10.

24. Lim G, Farrell LM, Facco FL, Gold MS, Wasan AD. Labor Analgesia as a Predictor for Reduced Postpartum Depression Scores: A Retrospective Observational Study. Anesth Analg. 2018;126(5):1598-605.

25. Sun J, Xiao Y, Zou L, et al. Epidural Labor Analgesia Is Associated with a Decreased Risk of the Edinburgh Postnatal Depression Scale in Trial of Labor after Cesarean: A Multicenter, Prospective Cohort Study. Biomed Res Int. 2020;2020:2408063.

26. Yang SN, Shen LJ, Ping T, Wang YC, Chien CW. The delivery mode and seasonal variation are associated with the development of postpartum depression. J Affect Disord. 2011;132(1-2):158-64.

27. Houston KA, Kaimal AJ, Nakagawa S, Gregorich SE, Yee LM, Kuppermann M. Mode of delivery and postpartum depression: the role of patient preferences. Am J Obstet Gynecol. 2015;212(2):229.e1-7. 
28. Gerli S, Fraternale F, Lucarini E, et al. Obstetric and psychosocial risk factors associated with maternity blues. J Matern Fetal Neonatal Med. 2019:1-6.

29. Farrar JT, Pritchett YL, Robinson M, Prakash A, Chappell A. The clinical importance of changes in the 0 to 10 numeric rating scale for worst, least, and average pain intensity: analyses of data from clinical trials of duloxetine in pain disorders. J Pain. 2010;11(2):109-18.

30. Lee LC, Hung $\mathrm{CH}$. Predictors of post-partum stress in Vietnamese immigrant women in Taiwan. Jpn J Nurs Sci. 2016;13(1):38-45.

31. Luthar SS, Becker BE. Privileged but pressured? A study of affluent youth. Child Dev. 2002;73(5):1593-610.

32. Adouard F, Glangeaud-Freudenthal NM, Golse B. Validation of the Edinburgh postnatal depression scale (EPDS) in a sample of women with high-risk pregnancies in France. Arch Womens Ment Health. 2005;8(2):89-95.

33. Dennis CL. Can we identify mothers at risk for postpartum depression in the immediate postpartum period using the Edinburgh Postnatal Depression Scale. J Affect Disord. 2004;78(2):163-9.

34. Jardri R, Pelta J, Maron M, et al. Predictive validation study of the Edinburgh Postnatal Depression Scale in the first week after delivery and risk analysis for postnatal depression. J Affect Disord. 2006;93(1-3):169-76.

35. Armony-Sivan R, Shao J, Li M, et al. No relationship between maternal iron status and postpartum depression in two samples in China. J Pregnancy. 2012;2012:521431.

36. Dlamini LP, Mahanya S, Dlamini SD, Shongwe MC. Prevalence and factors associated with postpartum depression at a primary healthcare facility in Eswatini. S Afr J Psychiatr. 2019;25(0):1404.

37. JayaSalengia B, Rajeswari S, Nalini S. The Relationship between Maternal Confidence, Infant Temperament, and Postpartum Depression. Iran J Nurs Midwifery Res. 2019;24(6):437-43.

38. Araújo IS, Aquino KS, Fagundes L, Santos VC. Postpartum Depression: Epidemiological Clinical Profile of Patients Attended In a Reference Public Maternity in Salvador-BA. Rev Bras Ginecol Obstet. 2019;41(3):155-63.

39. Farías-Antúnez S, Santos IS, Matijasevich A, de Barros A. Maternal mood symptoms in pregnancy and postpartum depression: association with exclusive breastfeeding in a population-based birth cohort. Soc Psychiatry Psychiatr Epidemiol. 2020.

40. Wang Z, Li C, Teng Y, et al. The Effect of lodine-Containing Vitamin Supplementation During Pregnancy on Thyroid Function in Late Pregnancy and Postpartum Depression in an lodine-Sufficient Area. Biol Trace Elem Res. 2020.

\section{Tables}

Table 1 Baseline characteristics of eligible patients 


\begin{tabular}{|c|c|c|c|}
\hline Parameters & Group LA $(n=97)$ & Group CS $(n=101)$ & $\begin{array}{l}P \\
\text { value }\end{array}$ \\
\hline Age $(y)$ & $27.88 \pm 3.34$ & $31.69 \pm 4.39$ & $\varangle 0.001$ \\
\hline $\mathrm{BMI}\left(\mathrm{kg} / \mathrm{m}^{2}\right)$ & $26.20 \pm 2.66$ & $27.34 \pm 2.95$ & $₫ 0.001$ \\
\hline Education $\otimes 12$ y & $86(88.65 \%)$ & $83(82.17 \%)$ & 0.197 \\
\hline Family income (10,000 CNY/month) & $0.83 \pm 0.29$ & $0.84 \pm 0.26$ & 0.790 \\
\hline Gravidity & $1(1-2)$ & $3(2-5)$ & $\varangle 0.001$ \\
\hline Parity & $0(0-0)$ & $1(0-1)$ & $\llbracket 0.001$ \\
\hline perinatal classes attending & $41(42.26 \%)$ & $19(18.81 \%)$ & $\llbracket 0.001$ \\
\hline $\begin{array}{l}\text { Present obstetrical and gynecological } \\
\text { disease }^{\text {a }}\end{array}$ & $49(50.51 \%)$ & $32(31.68 \%)$ & 0.015 \\
\hline Disease complicating pregnancy ${ }^{b}$ & $44(45.46 \%)$ & $63(62.37 \%)$ & 0.016 \\
\hline Antenatal depression & $21(21.64 \%)$ & $26(25.74 \%)$ & 0.499 \\
\hline Baseline NRS score & $7.38 \pm 1.26$ & $2.32 \pm 1.43$ & $\varangle 0.001$ \\
\hline
\end{tabular}

BMI: body mass index, NRS: numerical rating scale

Data were shown as mean $\pm S D$, median (range) or number of subjects (percentage)

${ }^{a}$ Including premature rupture of membranes, potential placenta implantation, placenta previa, oligohydramnion, colpitis, hysteromyoma, polycystic ovarian syndrome.

${ }^{b}$ Including diabetes, hypertension, anemia, thyroid dysfunction, streptococcal infection, respiratory infection, cholestasis, systematic lupus erythematosus (SLE), positive hepatitis B surface antigen.

Table 2 Perinatal variables of postpartum follow up 


\begin{tabular}{|c|c|c|c|}
\hline Parameters & Group LA (n=97) & Group CS $(n=101)$ & $P$ value \\
\hline \multicolumn{4}{|l|}{ NRS at rest after birth } \\
\hline 1 day & $2(1-3)$ & $2(1-3)$ & 0.598 \\
\hline 2 days & $1(0-2)$ & $1(0-1.5)$ & 0.058 \\
\hline 3 days & $0(0-0)$ & $0(0-0.5)$ & 0.001 \\
\hline 6 weeks & $0(0-0)$ & $0(0-0)$ & 0.869 \\
\hline \multicolumn{4}{|l|}{ NRS in motion after birth } \\
\hline 1 day & $3(2-5)$ & $4(3-6)$ & 0.002 \\
\hline 2 days & $2(1-4)$ & $2(2-4)$ & 0.743 \\
\hline 3 days & $1(1-2.5)$ & $1(1-2)$ & 0.631 \\
\hline 6 weeks & $0(0-0)$ & $0(0-1)$ & $\llbracket 0.001$ \\
\hline \multicolumn{4}{|l|}{ EPDS scores after birth } \\
\hline 3 days & $4(1-7)$ & $7(3-9)$ & 0.003 \\
\hline 6 weeks & $4(1-7)$ & $6(2-10)$ & 0.014 \\
\hline \multicolumn{4}{|c|}{ Adverse event after birth ${ }^{a}$} \\
\hline 3 days & $29(29.89 \%)$ & $18(17.82 \%)$ & 0.046 \\
\hline 6 weeks & $22(22.68 \%)$ & $31(30.69 \%)$ & 0.203 \\
\hline Duration of labor (min) & $691(581-823)$ & $40(35-47)$ & $\otimes 0.001$ \\
\hline Infant feeding & & & 0.099 \\
\hline Formula milk & $53(54.63 \%)$ & $49(48.51 \%)$ & \\
\hline Breast milk & $1(1.03 \%)$ & $7(6.93 \%)$ & \\
\hline Mixed feeding & $43(44.34 \%)$ & $45(44.56 \%)$ & \\
\hline Main companion & & & 0.01 \\
\hline Professional maid & $15(15.46 \%)$ & $25(24.75 \%)$ & \\
\hline Parents- in-law & $23(23.71 \%)$ & $31(30.69 \%)$ & \\
\hline Parturient's parents & $19(19.58 \%)$ & $32(31.68 \%)$ & \\
\hline Mixed companion & $40(41.25 \%)$ & $22(12.88 \%)$ & \\
\hline Occurrence of PPD & $11(11.34 \%)$ & $26(25.74)$ & 0.011 \\
\hline
\end{tabular}


NRS: numerical rating scale, EPDS: Edinburgh Postnatal Depression Scale, PPD: postpartum depression.

Data were shown as number of subjects (percentage) or median (range)

a adverse event included dizziness, low back pain, numbness of legs, uracratia, hydrosis, perineal lacerations, placenta remnant, nausea and vomiting, constipation, chest congestion, palpitation of participant, and jaundice, pneumonia, eczema, diarrhea, milk vomiting of neonate.

\section{Table 3 Neonatal variables of parturients}

\begin{tabular}{|llll|}
\hline Parameters & Group LA $(\mathrm{n}=97)$ & Group CS $(\mathrm{n}=101)$ & $P$ value \\
\hline Gender & & & 0.462 \\
\hline Male & $42(43.29 \%)$ & $49(48.51 \%)$ & \\
\hline Female & $55(56.71 \%)$ & $52(51.49 \%)$ & \\
\hline Body weight (g) & $3344.58 \pm 312.34$ & $3404.95 \pm 404.19$ & 0.242 \\
\hline Apgar score after birth & & & \\
\hline 1-min & $10(9-10)$ & $10(9-10)$ & 0.610 \\
\hline 5-min & $10(10-10)$ & $10(10-10)$ & 0.534 \\
\hline $10-$ min & $10(10-10)$ & $10(10-10)$ & 0.308 \\
\hline
\end{tabular}

Data were shown as mean \pm SD, median (range) or number of subjects (percentage)

Table 4 Univariate and multivariate regression analysis of PPD at 6 weeks after birth

\begin{tabular}{|c|c|c|c|c|}
\hline Parameters & Univariate & & Multivariate & \\
\hline & OR $(95 \% \mathrm{Cl})$ & $P$ & OR $(95 \% \mathrm{Cl})$ & $P$ \\
\hline Labor analgesia & $0.369(0.171-0.797)$ & 0.011 & $0.049(0.003-0.955)$ & 0.047 \\
\hline Family income & $5.365(1.598-18.004)$ & 0.007 & $7.267(1.65-32.014)$ & 0.009 \\
\hline Duration of labor & $0.999(0.998-1)$ & 0.088 & & \\
\hline Antenatal depression & $5.014(2.339-10.75)$ & $\varangle 0.001$ & & \\
\hline Perinatal classes attending & $2.103(0.867-5.101)$ & 0.1 & & \\
\hline Neonate of male & $1.907(0.922-3.943)$ & 0.082 & & \\
\hline EPDS at 3 days after birth & $1.281(1.17-1.402)$ & $\varangle 0.001$ & $1.275(1.14-1.426)$ & $\nabla 0.001$ \\
\hline $\begin{array}{l}\text { Adverse event at } 6 \text { weeks after } \\
\text { bitrh }\end{array}$ & $0.525(0.247-1.118)$ & 0.095 & & \\
\hline
\end{tabular}


NRS: numerical rating scale, EPDS: Edinburgh Postnatal Depression Scale, OR: odds ratio, Cl: confidence interval;

\section{Figures}

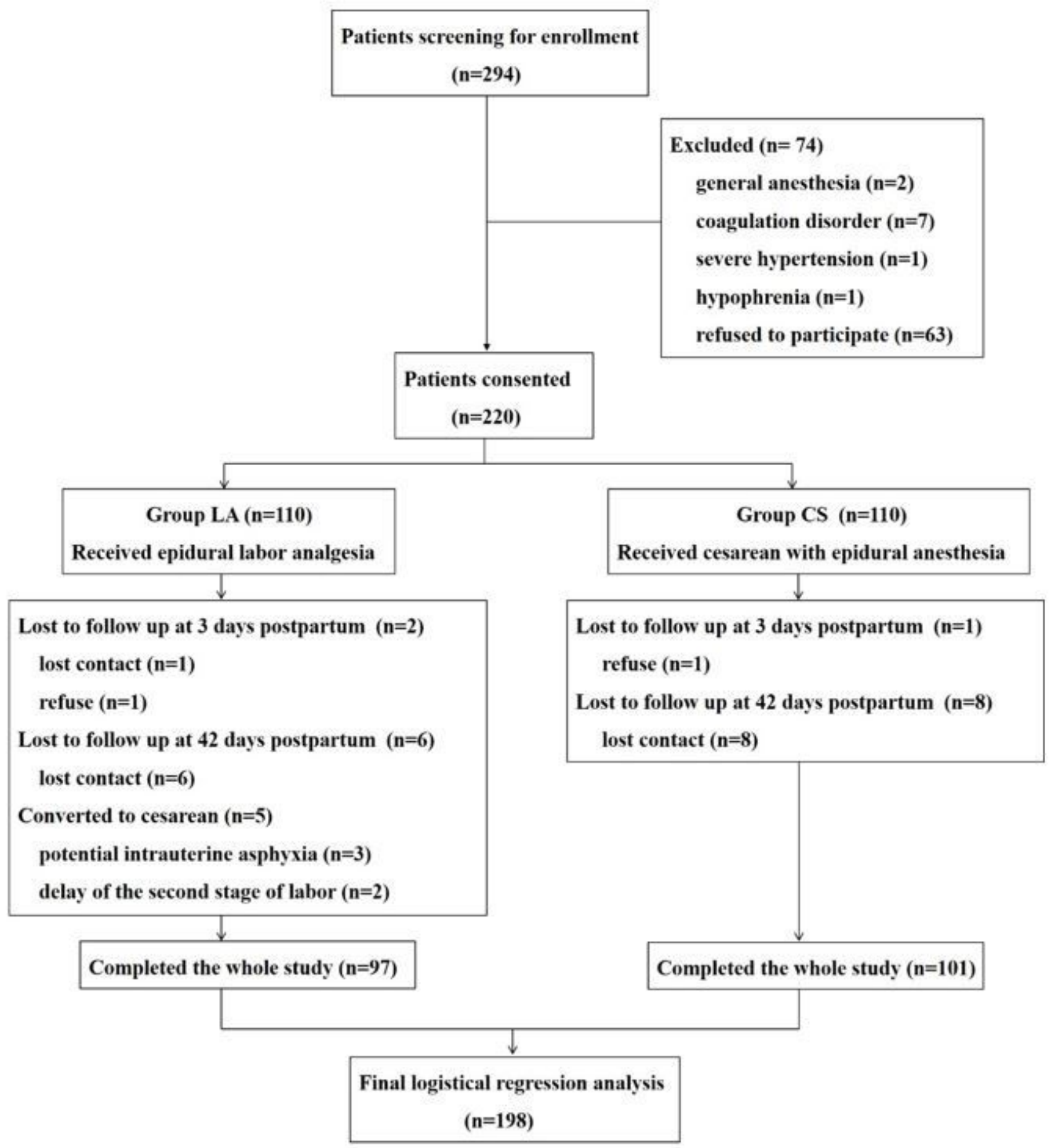

Figure 1. 


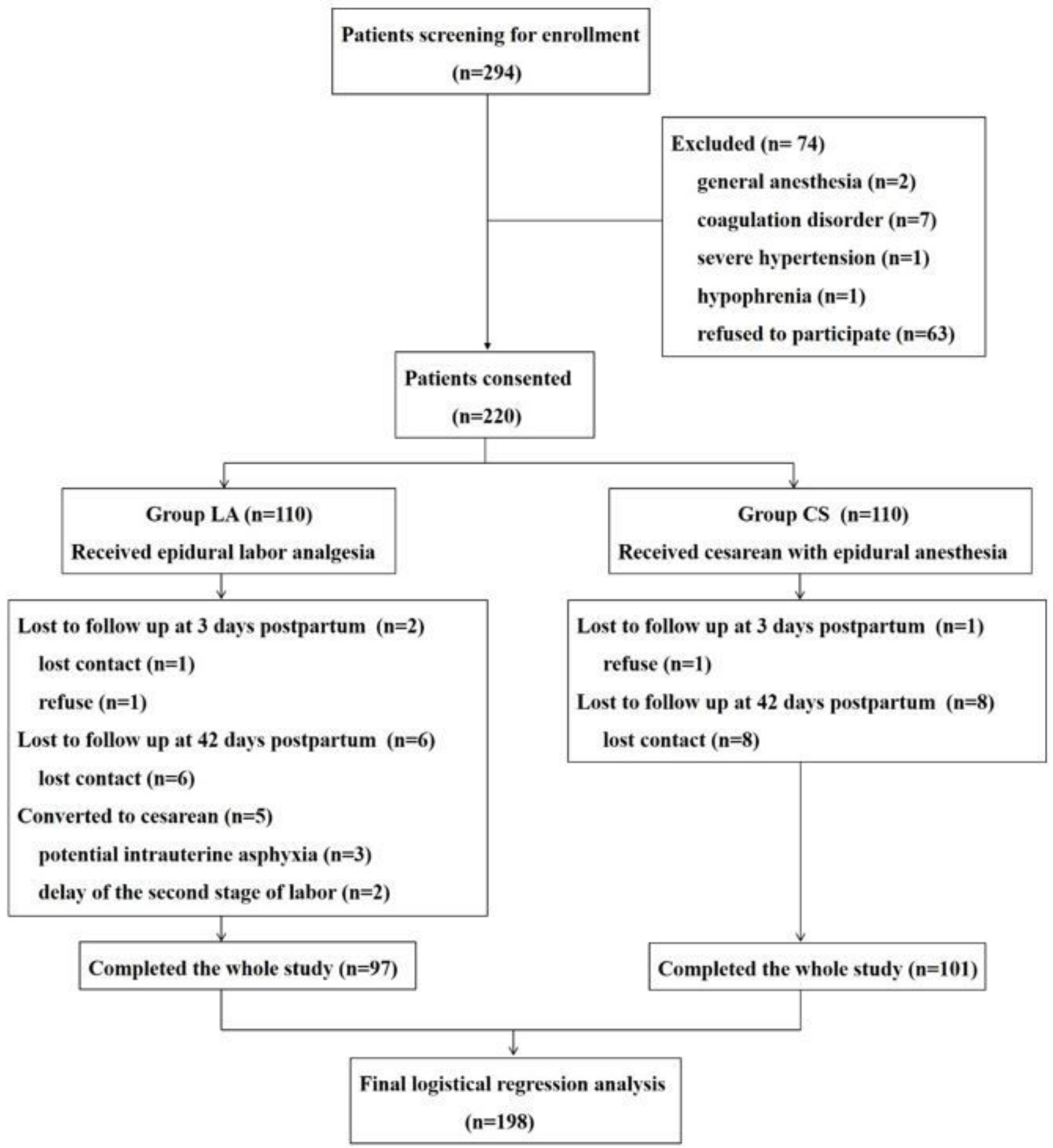

Figure 1.

\section{Figure 1}

Flow chart of the study 